\title{
Propiedades físicas de la madera de ejemplares jóvenes de Alnus glutinosa (L.) Gaertn.
}

Recibido: 30 setembro 2009 / Aceptado: 30 decembro 2009

(C) IBADER- Universidade de Santiago de Compostela 2010

\begin{abstract}
Resumen En el interior de Galicia (noroeste de España) se apeó un grupo de alisos jóvenes para caracterizar su madera analizando probetas sin defectos. Las propiedades físicas obtenidas presentaban una variación entre individuos altamente significativa. Los valores medios corresponden a una madera semipesada y medianamente estable (peso específico al $12 \%$ de humedad: $547 \mathrm{~kg} / \mathrm{m}^{3}$; densidad básica: $440 \mathrm{~kg} / \mathrm{m}^{3}$; contracción volumétrica: 14,3 $\%$; coeficiente de contracción volumétrica: 0,26 \%). La variación entre pies no es atribuible al medio, semejante para todos los árboles, ni a sus dimensiones, que no influyen significativamente en las propiedades de la madera. Solamente la edad del árbol influye significativamente en algunas características. Por tanto, la variación de la madera entre individuos obedece a causas hereditarias, quedando justificado que esta especie sea una fuente de diversidad genética a conservar. Se propone un modelo que describe la variación de la densidad básica a lo largo del fuste $\left(R^{2}=\right.$ $0,62 ; E=6 \cdot 10-5 \% ; R M S E=5,1 \%)$. La validez del modelo para todos los individuos muestreados y para los seis primeros metros de fuste permite calcular, a partir del volumen de corta, la materia seca que se obtendría al aprovechar las dos o tres primeras trozas comerciales.
\end{abstract}

Palabras clave aliso ' peso específico ' densidad básica contracción.

Guillermo Riesco Muñoz · Ana María Rodríguez Campos Departamento de Enxeñería Agroforestal, Universidade de Santiago de Compostela.

Escola Politécnica Superior. Campus Universitario s/n. 27002 Lugo (España).

Tel: 982-285900; Fax: 982-285926

e-mail: guillermo.riesco@usc.es
Summary A group of young alders was felled in a remote area of Galicia (northwest Spain), for analysis of wood properties. The following mean values of different properties were obtained on small defect-free specimens of wood (airdry density: $547 \mathrm{~kg} / \mathrm{m}^{3}$; basic density: $440 \mathrm{~kg} / \mathrm{m}^{3}$; volumetric shrinkage: $14.3 \%$; volumetric shrinkage coefficient: $0.26 \%$ ). The wood was moderately heavy and stable. Highly significant inter-tree variation was observed for all properties considered. This variation cannot be attributed to environmental conditions, which were almost constant for all sampled trees. The variations in the characteristics of the wood were not significantly affected by the tree dimensions. Only the age of the trees significantly affected the mean values of some wood properties. Therefore, the inter-tree variation in wood properties was mainly due to genetic causes, which suggests that this species should be protected as a source of tree diversity. A model is proposed for predicting the variation in basic density along the stem $\left(R^{2}=0.62 ; E=6 \cdot 10-5 \% ; R M S E=5.1 \%\right)$. The general validity of the model for all sampled individuals up to six meters in the stems enables calculation of the amount of dry matter in the two or three lower commercial logs, from the harvested volume.

Key words alder · specific gravity · basic density · shrinkage

\section{Introducción}

El aliso, Alnus glutinosa (L.) Gaertn., es una betulácea que se distribuye de forma natural por Europa, Siberia y el noroeste de África. Aparece en toda España pero es más frecuente en el cuadrante noroeste. En Galicia aparece desde el nivel del mar hasta los $1200 \mathrm{~m}$ de altitud (Álvarez et al. 2000), de forma aislada, formando pequeños bosquetes o formando bosques en galería en las riberas de los ríos, en los sotos y en las vaguadas. Se suele presentar en mezcla con otras frondosas y tiene su estación propia en suelos húmedos o temporalmente encharcados, prefiriendo los sueltos y fértiles. Es una especie heliófila y mejoradora, 
capaz de colonizar suelos rocosos y carentes de materia orgánica, gracias a su capacidad para fijar el nitrógeno atmosférico (Ruiz de la Torre 1979).

El aliso alcanza poca altura, unos $12 \mathrm{~m}$, aunque puede llegar a los 30 m (Collardet \& Besset 1992; Nájera et al. 1969; Ruiz de la Torre 1979). Su longevidad no suele pasar de 100 años y la edad de corta habitual se encuentra entre los 30 y los 50 años. Al final del turno puede presentar $25 \mathrm{~m}$ de altura media y un diámetro normal de $30 \mathrm{~cm}$, con producciones entre 9 y $11 \mathrm{~m}^{3} /$ ha/año (Álvarez et al. 2000).

El porte de los fustes es derecho y cilíndrico, sin bifurcaciones y con buena poda natural. La madera es de calidad media, ligera y blanda. Es estable ante los cambios de humedad, poco durable a la intemperie aunque muy durable si se mantiene sumergida (Ruiz de la Torre 1979). Es madera de fibra recta, homogénea, en la que resulta difícil distinguir la albura del duramen. Presenta los anillos de crecimiento anual bien marcados, con una anchura de anillo en torno a 3,3 $\mathrm{mm}$ y con la madera de verano más oscura. Es fácil de trabajar, secar y teñir. Debido a esto y a su densidad, similar a la de la caoba, es adecuada para imitar a ésta y a otras maderas de calidad (ébano, nogal, cerezo). Se emplea mucho en objetos tallados o torneados y tradicionalmente se usaba en obras hidráulicas (Kollmann 1959). La leña es de baja calidad pero su carbón se empleaba para fabricar pólvora negra. Las lupias y verrugas se destinan a la obtención de chapa decorativa (Nájera et al. 1969).

España es el país de la Unión Europea que más madera de frondosas importa de Estados Unidos. La tercera madera que más se importa es el aliso (Alnus rubra, Alnus incana), de la que entraron en nuestro país unos $50.000 \mathrm{~m}^{3}$ con corteza en 2004, con una tendencia al aumento de la importación (ACEMM 2005). Se importa, así mismo, madera de aliso de Centroeuropa (Alnus glutinosa), aunque la madera de esta especie sufre más grietas de secado que la de Alnus rubra (Johnson 1989).

A pesar de la demanda de la industria y del valor ambiental del aliso en los ecosistemas ripícolas, las alisedas han sido frecuentemente reemplazadas por cultivos agrícolas, choperas o prados de siega. El desinterés de los propietarios forestales hacia la conservación del aliso se puede explicar en parte por el bajo precio de venta de esta madera, similar al de la madera de pino de igual dimensión, y muy inferior al precio que alcanzan otras frondosas caducifolias de producción nacional (Álvarez et al. 2000).

Otros factores contribuyen, así mismo, a la degradación de las alisedas: la fragilidad de estas masas debido a su habitual fragmentación, la canalización de los cauces fluviales, la construcción de embalses, el ajardinamiento de las riberas, los drenajes o la extracción de áridos (Rigueiro Rodríguez 2003). A estos factores hay que añadir más recientemente un debilitamiento severo de la especie, que se observa desde los años 90, acentuándose en los últimos años con una elevada mortalidad del aliso en Galicia y en otras regiones de España. Se trata de una mortalidad específica, que no afecta a otras especies que pueden compartir con el aliso el mismo nicho ecológico, como el sauce, el arce, el abedul o el fresno. Aunque el origen de la mortalidad se atribuye a un hongo del género Phytophthora las determinaciones efectuadas sobre muestras de pies enfermos en Galicia solo han permitido detectar hongos del género Armillaria. A escala continental, se ha citado la presencia de Phytophthora en alisedas de Francia, Bélgica e Inglaterra (Cavelier et al. 1999; Gibbs et al. 1999) así como en Polonia y Alemania (Orlikowski \& Oszako 2005).

Ante dichos factores, que amenazan la pervivencia del aliso, se ha propuesto la conservación en Europa del patrimonio genético de la especie (Kajba \& Gracan 2003), cuya importancia y diversidad ha sido referida por otros autores (De Wald et al. 1983; Genys 1988; Prat et al. 1992). Cualquier medida de conservación de la diversidad genética del aliso parte de su caracterización, incluyendo el estudio de las propiedades de su madera. En España no se han publicado estudios sobre la madera de aliso, salvo el trabajo de referencia de Gutiérrez Oliva \& Plaza Pulgar (1967), y a nivel europeo escasea la información publicada sobre madera de aliso joven, aunque la especie sea objeto de aprovechamiento maderero tanto al llegar a la madurez como en edades juveniles.

Las propiedades de la madera que merecen mayor interés son las propiedades gravimétricas (peso específico y densidad) por tratarse de indicadores habituales de calidad en la madera. Así mismo, debe cuantificarse la estabilidad de la madera mediante las variables contracción volumétrica, coeficiente de contracción volumétrica e higroscopicidad, ya que el empleo de la madera viene condicionado por su estabilidad dimensional ante los cambios de humedad en el ambiente. Por último, la madera juvenil, más inestable, abunda en ejemplares jóvenes, lo que limita el aprovechamiento de éstos. Para detectar la presencia de madera juvenil se estudia la contracción de la madera en la dirección de la fibra (contracción longitudinal y coeficiente de contracción longitudinal), ya que dichas variables presentan valores elevados y gran dispersión en la madera juvenil (Riesco Muñoz 2001).

El objetivo general del presente estudio es analizar las propiedades físicas de la madera de alisos jóvenes procedentes de Galicia y sin síntomas aparentes de decaimiento por hongos.

\section{Material y métodos}

Se procedió a la selección y apeo de 5 ejemplares aparentemente sanos de la especie Alnus glutinosa (L.) Gaertn. en una parcela situada en Bacurín, término municipal de Lugo, en el interior de Galicia (x: 606912; y: 4758235; altitud: $525 \mathrm{~m}$ ). El número de árboles elegido cubría las exigencias de la norma española UNE 56528:1978 y de la norma europea EN 384:2004. Se localizó la parcela mediante sus coordenadas geográficas con ayuda de un navegador GPS con precisión de \pm 5 ". La altitud se calculó con un altímetro barométrico de $\pm 5 \mathrm{~m}$ de precisión. El diámetro normal se midió con forcípula de $\pm 0,5$ $\mathrm{cm}$ de precisión y la altura total con hipsómetro Blume-Leiss con precisión de $\pm 0,5 \mathrm{~m}$. La edad de cada fuste se 
determinó en laboratorio por conteo de anillos sobre las rodajas basales. La esbeltez de cada pie se obtuvo como cociente entre altura total y diámetro normal (Tabla 1).

De cada aliso apeado se obtuvieron rodajas de la sección transversal completa del fuste a distancias fijas de un metro, empezando desde la base y hasta $6 \mathrm{~m}$ de altura en el tronco. Las rodajas se cortaron con un espesor aproximado de $10 \mathrm{~cm}$ y fueron labradas en carpintería hasta obtener probetas prismáticas de 2 por 2 por $4 \mathrm{~cm}$ como dimensiones nominales en la dirección radial, tangencial y longitudinal respectivamente. Sobre las probetas de ensayo que resultaron libres de defectos anatómicos, con desviación de fibra inapreciable, con anillos de crecimiento paralelos a las aristas y sensiblemente rectos (no muy próximos a la médula), se determinaron las propiedades físicas que aparecen en la Tabla 2. Dichas variables se obtuvieron según disponen las normas españolas UNE 56528:1978, UNE 56531:1977, UNE 56532:1977, UNE 56533:1977 y según las normas europeas EN 13183-1:2002 y EN 408:2003. El peso de cada probeta se obtuvo con precisión de centésima de gramo y el volumen se obtuvo considerando cada pieza como un prisma perfecto y multiplicando sus tres dimensiones principales, obtenidas con precisión de centésima de milímetro. El análisis sobre probetas de pequeñas dimensiones sin defectos permite caracterizar la madera de una especie y comparar madera de diversas procedencias ya que la presencia de defectos o singularidades en la madera es una fuente de variación que impediría detectar la influencia de otros factores en las propiedades del material.

\begin{tabular}{|c|c|c|c|c|c|}
\hline$n^{\circ}$ & $\begin{array}{l}\text { eded } \\
\text { (arros) }\end{array}$ & $\begin{array}{c}\text { ¿ ciametro nomal con corteza } \\
\text { (cm) }\end{array}$ & $\begin{array}{c}\text { is a ture totel } \\
\text { (min) }\end{array}$ & $\begin{array}{l}\text { esbelbez } \\
\text { (Fid) }\end{array}$ & $\begin{array}{c}\text { credmiento racisic.c. } \\
\text { (mervario) }\end{array}$ \\
\hline 1 & 18 & 11,5 & 11,9 & 1,04 & 3,2 \\
\hline 2 & 20 & 11,0 & 11,1 & 1,01 & 2,8 \\
\hline 3 & 22 & 11,0 & 14,8 & 1.35 & 2,5 \\
\hline 4 & 24 & 13,9 & 14,4 & 1,04 & 2,9 \\
\hline 5 & 25 & 13.0 & 12.7 & 0.98 & 2,5 \\
\hline
\end{tabular}

Tabla 1.- Características dendrométricas de los alisos de la muestra. c.c. = con corteza

Tras validar y depurar los resultados de los ensayos se obtuvieron los estadísticos descriptivos básicos de las propiedades físicas (Tabla 2). Para encontrar relaciones entre variables cuantitativas y cualitativas se efectuó el análisis de la varianza con un factor. Se hizo uso de técnicas de regresión para presentar y describir relaciones de interés entre variables cuantitativas. Entre las variables que presentaron valores significativos del coeficiente de correlación lineal de Pearson $(R)$ se buscaron modelos, por el método de ajuste de mínimos cuadrados ordinarios, que permitieran estimar una variable como función de otras variables independientes, no correlacionadas linealmente entre sí. Como criterios de selección de modelos se emplearon el coeficiente de determinación $\left(R^{2}\right)$, el sesgo en porcentaje $(E)$ y la raíz del error medio cuadrático en porcentaje (RMSE).

En todas las pruebas estadísticas se consideró que un nivel de significación bilateral menor o igual a 0,05 es significativo y que un valor menor o igual a 0,01 es altamente significativo.

\begin{tabular}{|c|c|c|c|c|}
\hline & minms & maxomo & meda & $C V(\$)$ \\
\hline anctura ce arillos (mm) & 20 & 5,1 & 3,4 & 22,2 \\
\hline humedad $(\%)$ & 4,8 & 13,5 & 9,4 & 10,6 \\
\hline humadad náxima tabrice (\%) & 1284 & 202,2 & 164.9 & 11.2 \\
\hline peso especifico at $12 \%$ de humeckad (kgimi) & 458 & 683 & 547 & 8,4 \\
\hline pere especifico anhidro \{kght & 423 & 611 & 502 & 8,5 \\
\hline densidad barsica $\left(\mathrm{kg}^{\prime} \mathrm{m}^{2}\right)$ & 375 & 525 & 440 & 0,2 \\
\hline contraccion volu nátrica (s) & 4,7 & 20,9 & 14,3 & 19,2 \\
\hline cceficiente de contracción volumetrica $(\%)$ & 0.01 & 1,00 & 0.26 & $7 \Theta, 3$ \\
\hline higroscopicidad ; $k g i m)$ & 0,0 & $\$, 93$ & 4.06 & 27.7 \\
\hline controcetion longitudina (98) & 0,0 & 2,2 & 0,7 & 51.8 \\
\hline cceficiente de contraccón long tudinal (\%) & 000 & 0,12 & 0,03 & 73.3 \\
\hline congtants ce Kcehiar wolu netrica & 92 & 36.4 & 28,6 & 18.4 \\
\hline constarye ce Kcehier longitudinal & 0.1 & 4.2 & 1,3 & 50.4 \\
\hline
\end{tabular}

Tabla 2.- Estadísticos descriptivos de las propiedades físicas analizadas en la madera de aliso $(n=221$ probetas). $C V(\%)=$ coeficiente de variación 


\section{Resultados y discusión}

\section{Estadísticos descriptivos básicos}

La muestra está constituida por alisos jóvenes, que están completando la primera mitad de la rotación habitual de la especie (Tabla 1). Se ensayaron en promedio 44 probetas por pie, con un mínimo de 20 probetas en un ejemplar. La Tabla 2 muestra los principales estadísticos de las propiedades físicas analizadas. Se trata de una madera semipesada, medianamente estable y muy higroscópica, con una estabilidad dimensional normal para la densidad que presenta. La anchura media de los anillos anuales de crecimiento (Tabla 1 y Tabla 2) toma un valor usual en la especie. Destaca que la humedad de equilibrio higroscópico alcanzada por la madera en las condiciones higrométricas de laboratorio es, en promedio, muy inferior al $12 \%$, que es la humedad de equilibrio habitual en otras frondosas como el roble (Riesco Muñoz 2001). La media obtenida para el peso específico al $12 \%$ de humedad es un valor intermedio entre los que refiere la bibliografía (Álvarez et al. 2000; Collardet \& Besset 1992; Gutiérrez Oliva \& Plaza Pulgar 1967; Nutsch 1992; Peraza Oramas 1976; Ruiz de la Torre 1979; Vignote Peña \& Martínez Rojas 2006; Vignote Peña et al. 2000; Wagenführ \& Scheiber 1974). Según el criterio de interpretación dado por la norma UNE 56540:1978 para madera de frondosas, el aliso queda dentro del rango de las maderas semipesadas. El peso específico anhidro es un 4 \% inferior al citado por Wagenführ \& Scheiber (1974) en tanto que la densidad básica es un $9 \%$ superior a la obtenida por Gutiérrez Oliva \& Plaza Pulgar (1967).

La contracción volumétrica toma un valor superior al más alto que ofrece la bibliografía consultada, que es la referencia de Vignote Peña et al. (2000). Sin embargo, la constante de Koehler (1931) se encuentra en torno al valor teórico, 28, lo que indica que la contracción volumétrica es la esperable para el peso específico de la madera. De hecho, la contracción volumétrica es de tipo medio según el criterio de interpretación dado por la norma UNE 56540:1978. El coeficiente de contracción volumétrica toma un valor intermedio entre los que refieren los trabajos consultados (Collardet \& Besset 1992; Gutiérrez Oliva \& Plaza Pulgar 1967; Vignote Peña \& Martínez Rojas 2006;
Vignote Peña et al. 2000; Wagenführ \& Scheiber 1974) y es el propio de una madera estable ante los cambios de humedad y fuertemente higroscópica (criterio de interpretación de la norma UNE 56540:1978). La contracción longitudinal es más alta que la citada por Wagenführ \& Scheiber (1974) pero es la esperable para el peso específico que presenta la madera (constante de Koehler próxima al valor teórico de 1,5) y para individuos que aún no han llegado a la madurez, en los que abunda la madera juvenil, que presenta contracción longitudinal elevada.

La dispersión de los resultados, dada por el coeficiente de variación, es inferior al $30 \%$ en la mayor parte de las variables, a excepción del coeficiente de contracción volumétrica y de las variables relacionadas con la estabilidad de la madera en dirección longitudinal (contracción, coeficiente y constante de Koehler). La alta dispersión de estas variables es previsible dado que la madera procede de pies jóvenes, en los que hay alta proporción de madera juvenil, en la cual las propiedades de contracción presentan gran variabilidad (Riesco Muñoz 2001).

El error relativo en la estimación de la media es inferior al 5 $\%$, umbral establecido por la norma UNE 56528:1978, para todas las variables salvo para aquéllas que se acaban de subrayar por presentar un coeficiente de variación superior a $30 \%$.

\section{Relaciones entre propiedades físicas de la madera}

La anchura de anillos de crecimiento y la densidad de la madera suelen emplearse como indicadores de calidad en la madera por su vinculación con otras propiedades de la misma. Sin embargo, en la muestra analizada no aparecieron correlaciones destacables entre la anchura de anillos, peso específico o densidad y el resto de propiedades físicas de la madera. La correlación más destacada $\left(R=0,25^{\star \star}\right)$ es la que se da entre contracción volumétrica y peso específico anhidro (Tabla 3). No aparecen mayores correlaciones cuando se analiza por separado la submuestra correspondiente a cada árbol de forma individual.

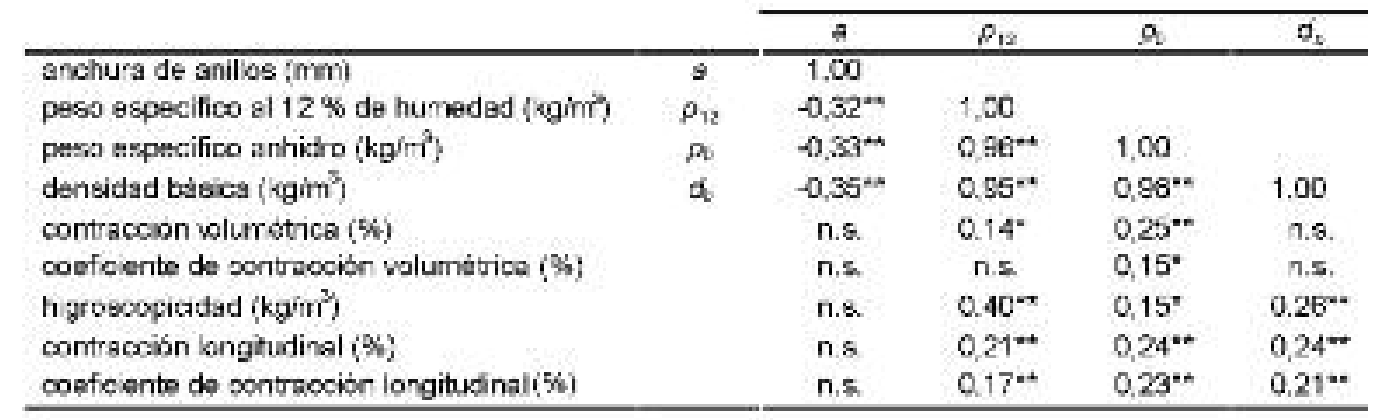

Tabla 3.- Coeficientes de correlación de Pearson $(R)$ entre las propiedades físicas analizadas en la madera de aliso (Alnus glutinosa) 
Variación de las características de la madera entre árboles

Para comprobar la influencia de las características de los árboles sobre las propiedades físicas de la madera se efectuó el correspondiente análisis de la varianza con un factor, resultando que todas las variables analizadas en la madera presentaban una variación altamente significativa entre individuos $(p<0,01)$. Dado que no hay diferencias de estación destacables entre árboles, la variación entre pies podría atribuirse en principio a las diferentes características de los mismos (edad, diámetro normal, altura total, esbeltez, anchura media de anillos de crecimiento). Se comprobó, sin embargo, que solamente había correlación significativa entre el peso específico medio del árbol y su edad:

$$
\begin{aligned}
& R=0,96^{* *} \text { entre peso específico al } 12 \% \text { y edad } \\
& R=0,93^{*} \text { entre peso específico anhidro y edad } \\
& R=0,77 \text { entre densidad básica y edad }
\end{aligned}
$$

En la Figura 1 se presenta la variación del peso específico al $12 \%$ en función de la edad del árbol y en función de la altura en el fuste. Debido a la elevada correlación entre peso específico medio por árbol y edad puede considerarse una vinculación lineal y positiva entre dichas variables $\mathrm{y}$, extrapolando, a los 30 años de edad el peso específico al 12 $\%$ sería, en promedio, de $581 \mathrm{~kg} / \mathrm{m}^{3}$. Es decir, a una edad adecuada para la corta la madera continuaría dentro del rango de las maderas semipesadas y alcanzaría un peso específico similar al citado por Álvarez et al. (2000) para ejemplares adultos.

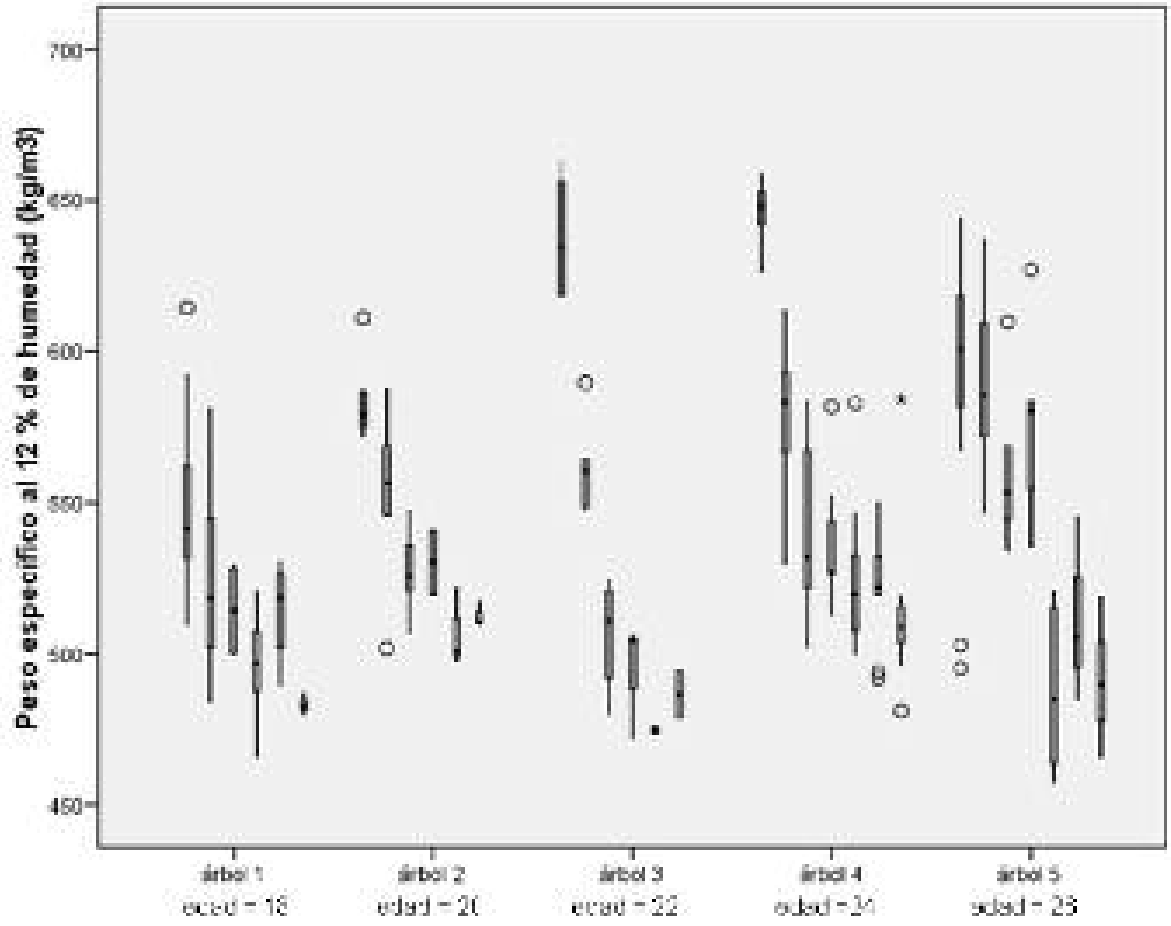

Figura 1.- Distribución de la variable peso específico al $12 \%$ en la madera de aliso. Las cajas representan la distribución de la muestra a cada altura en el fuste para cada árbol. Los árboles están ordenados por edad creciente
Variación de las características de la madera en el fuste

La mayor parte de las propiedades físicas analizadas presentan una variación altamente significativa a lo largo del fuste (coeficientes de correlación de Pearson en torno a $0,7^{* *}$ para las variables gravimétricas y en torno a $-0,2^{* *}$ para la higroscopicidad y para las variables de contracción longitudinal). Las correlaciones son algo mayores ( $R$ entre $0,62^{* *}$ y $\left.-0,86^{\star *}\right)$ cuando se analiza la submuestra de cada árbol por separado.

En el caso de la densidad básica, la correlación con la posición en el fuste mejora cuando se considera la altura relativa en el fuste (altura expresada como porcentaje de la altura total del árbol) en lugar de la altura en el fuste (expresada en metros). La variación de densidad básica a lo largo del fuste para la muestra completa se puede observar en la Figura 2. No obstante, el factor árbol y la posición en el fuste solo explican una parte de la variación de la densidad básica. De hecho, el coeficiente de variación de la densidad básica en cada árbol y para cada altura en el fuste (submuestra correspondiente a una rodaja) no es un valor despreciable (coeficiente de variación en torno a 3,2 \%). Se ha comprobado que la anchura de anillos no tiene una influencia destacable, y aprovechable con valor predictor, en dicho coeficiente de variación ya que solo en tres de las 32 rodajas analizadas existía correlación significativa entre densidad básica y anchura de anillos.

Se escogieron cuatro modelos (Tabla 4) que describían con precisión suficiente la relación que existe entre densidad 
básica y altura relativa en el fuste:

$$
\begin{aligned}
& d_{2}=493.200-5,838 h+0,194 m^{2}-0,002 i^{2} \\
& \alpha_{\mathrm{t}}=406,857-20,877 \mathrm{nn}\left(\frac{75,850}{\mathrm{~h}}-1\right) \\
& t_{s}=501,561-25,181 \mathrm{r} / \mathrm{w} \text {, } \\
& d_{0}=450,300-4,316 \mathrm{dr}+00.054 \mathrm{~m}^{2}
\end{aligned}
$$

Dadas las diferencias entre individuos señaladas en el apartado anterior se ajustaron los modelos para cada árbol individual, con lo que mejoraron los ajustes: valores de $R^{2}$ en torno a 0,70 para el modelo (1). Sin embargo, los coeficientes obtenidos para estos modelos individuales no eran predecibles con suficiente exactitud a partir de las características dendrométricas de los fustes (edad, diámetro normal, altura) por lo que se trataba de modelos sin utilidad práctica. Por ello, se optó por ajustar un modelo conjunto para todos los ejemplares de la muestra.

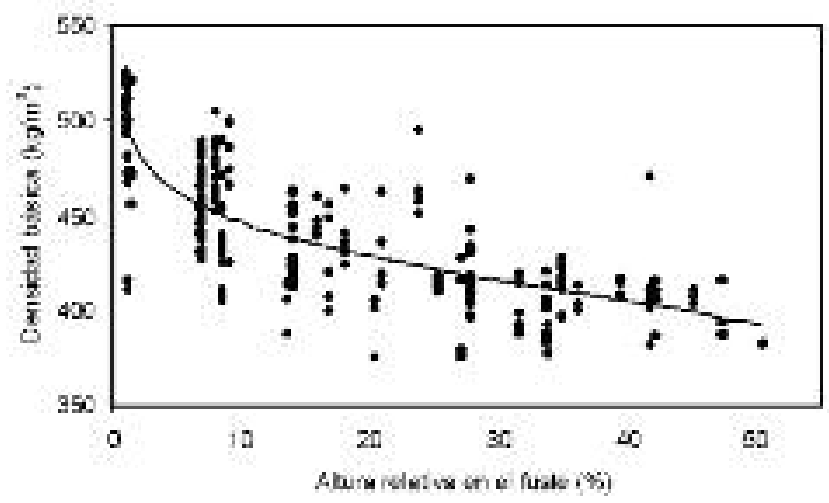

Figura 2.- Variación de la densidad básica de la

\begin{tabular}{|c|c|c|c|c|c|c|c|}
\hline ecuación & $F$ & $\begin{array}{l}\text { núroro de } \\
\text { parámotros }\end{array}$ & $\begin{array}{l}\text { grachos de } \\
\text { litertad }\end{array}$ & significación & $R^{2}$ & $\underset{(\&)}{E}$ & $\begin{array}{c}\text { RMSE } \\
(\%)\end{array}$ \\
\hline (1) & 118,785 & 4 & 217 & 0,000 & 0,62 & $-0,053331$ & 5,00 \\
\hline (2) & 354,699 & 3 & 219 & 0,000 & 0,62 & 0,000081 & 5.10 \\
\hline (3) & 341,590 & 2 & 219 & 0,000 & 0,61 & 0,000191 & 5,15 \\
\hline (4) & 165,612 & 3 & 218 & 0,000 & 0.60 & -0.021221 & 5.20 \\
\hline
\end{tabular}
madera de aliso a lo largo del fuste y modelo de predicción basado en la ecuación Verhulst-logística (Verhulst 1838)

Tabla 4.- Resultado del ensayo de modelos para describir la evolución de la densidad básica de la madera de aliso a lo largo del fuste

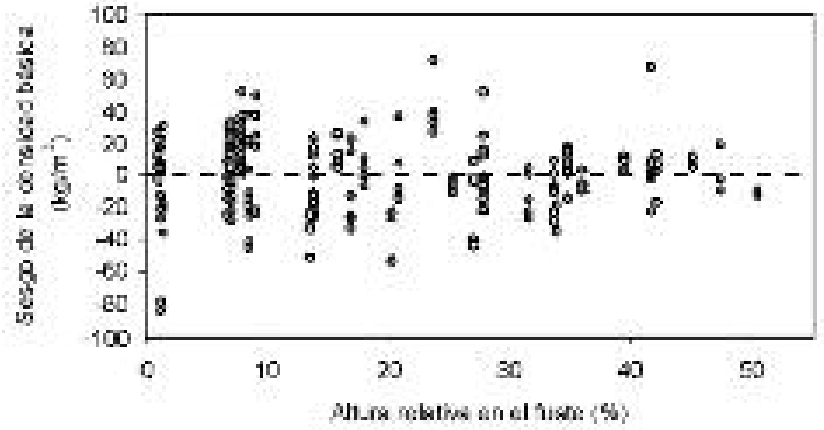

Figura 3.- Sesgo en la estimación de la densidad básica de la madera de aliso a lo largo del fuste con el modelo de predicción basado en la ecuación Verhulst-logística (Verhulst 1838)
El estudio del coeficiente de determinación, sesgo y residuo medio cuadrático de los modelos permitió elegir la ecuación (2) como la más adecuada para describir la tendencia de la densidad básica a lo largo del fuste. En efecto, todos los modelos presentaban unos valores de $R^{2}$ y de $R M S E$ muy similares pero el sesgo era drásticamente inferior en el modelo (2). No es el modelo más sencillo de los ensayados, ya que es triparamétrico y no linealizable. Sin embargo, el análisis de la nube de puntos (Figuras 2 y 3 ) reafirma la elección del modelo (2) ya que en la parte superior de los fustes (alturas superiores a unos $4 \mathrm{~m}$ ) los demás modelos tendían a sobreestimar la densidad básica. La expresión matemática del modelo (2) es la función inversa de la curva Verhulst-logística (Verhulst 1838), cambiando el signo de la variable independiente. Según el modelo ajustado, entre la base del árbol y la mitad de su altura $(6 \mathrm{~m}$ de altura en promedio) se produce una reducción de $104 \mathrm{~kg} / \mathrm{m}^{3}$ en la densidad básica. Se trata de una importante reducción, un 
$24 \%$ respecto de la densidad básica media de la madera. De dicha reducción, casi la mitad $(10 \%)$ se produce en el primer metro de altura de fuste, tal como se observa por la acusada pendiente inicial de la curva que representa el modelo (Figura 2). Desde el punto de vista del aprovechamiento del recurso, para incrementar la cantidad de materia leñosa seca extraída por unidad de volumen debería aprovecharse en el monte solo la primera troza comercial (hasta 2-2,5 m de altura en el fuste) debido a la reducida densidad de las trozas superiores.

\section{Conclusión}

La madera de aliso presenta densidad media y es moderadamente estable. Las propiedades físicas varían significativamente entre individuos, lo que constituye una evidencia de la diversidad que presenta la especie en Galicia y conduce a la necesidad de conservar las alisedas en todo el territorio gallego. Se ha modelizado la variación de la densidad básica a lo largo del fuste por lo que es posible calcular con suficiente precisión la cantidad de materia seca que presentaría una troza de volumen y posición en el fuste conocidos. La mayor parte de la materia leñosa seca se acumula en la parte inferior del fuste por lo que es más aconsejable el aprovechamiento comercial de las trozas basales.

Agradecimientos Para la realización de este trabajo se ha dispuesto de financiación procedente del Plan Gallego de Investigación, Desarrollo e Innovación Tecnológica (código de proyecto PGIDIT06RFO005E), que permitió ejecutar un contrato de investigación industrial para la empresa Industrias del Tablero S.A. (código de contrato 2006/CG528). Se agradece su colaboración en este trabajo a Don Santiago García Sánchez.

\section{Bibliografía}

ACEMM (2005). España sigue siendo el principal mercado de la Unión Europea (15) para la madera de frondosas estadounidenses. ACEMM. 17: 41-43.

Álvarez, P., Barrio, M., Díaz, R.A., Higueras, J., Riesco, G., Rigueiro, A., Rodríguez, R.J. \& Villarino, J.J. (2000). Manual de selvicultura de frondosas caducifolias. Serie Manuales Técnicos. Proyecto Columella, Lugo.

Cavelier, M., Claessens, H. \& Etienne, M. (1999). Premier signalement du Phytophthora de l'aulne (Alnus glutinosa) en Belgique. Parasitica. 55, 2-3 : 63-71.

Collardet, J. \& Besset, J. (1992). Bois commerciaux (vol. II). Feuillus des zones tempérées. Ed. H. Vial y CTBA.

De Wald, L.E., Steiner, K.C. \& Carter, K.K. (1983). Juvenile performance in three black alder provenance plantations in the northeast. Proc. Northeast. Forest Tree Improv. 28: 3739.

Genys, J.B. (1988). Intraspecific variation among 28 different sources of black alder, Alnus glutinosa (Betulaceae). Castanea. 53: 71-79.
Gibbs, J.N., Lipscombe, M.A. \& Peace, A.J. (1999). The impact of Phytophthora disease on riparian populations of common alder (Alnus glutinosa) in southern Britain. Eur. J. For. Path. 29: 39-50.

Gutiérrez Oliva, A. \& Plaza Pulgar, F. (1967). Características físico-mecánicas de las maderas españolas. Instituto Forestal de Investigaciones y Experiencias, Madrid.

Johnson, H. (1989). La madera. Blume, Barcelona.

Kajba, D. \& Gracan, J. (2003). Black alder (Alnus glutinosa). Bioversity. IPGRI, Roma.

Koehler, A. (1931). Longitudinal shrinkage of wood. Transactions of the American Soc. of Mechanical Engineers. 53, 2: 17-20.

Kollmann, F. (1959). Tecnología de la madera y sus aplicaciones. Vol. I. IFIE, Madrid.

Nájera y Angulo, F. \& López Fraile, V. (1969). Estudio de las principales maderas comerciales de frondosas peninsulares. Instituto Forestal de Investigaciones y Experiencias, Madrid.

Nutsch, W. (1992). Tecnología de la madera y del mueble. Reverté S. A., Barcelona.

Orlikowski, L.B. \& Oszako, T. (2005). Phytophthora cambivora on Alnus glutinosa: isolation and colonisation of plants. Journal of Plant Protection Research. 45, 4: 267-272.

Peraza Oramas, C. (1976). La madera y sus propiedades. Boletín de Información Técnica de AITIM. 80: 2-26.

Prat, D., Leger, C. \& Bojovic, S. (1992). Genetic diversity among Alnus glutinosa (L.) Gaertn. populations. Acta Oecol. 13, 4: 469-477.

Riesco Muñoz, G. (2001). Estudio de las propiedades físicomecánicas de la madera de roble (Quercus robur L.) de Galicia en relación con las variables del medio. Tesis Doctoral. Universidad Politécnica de Madrid.

Rigueiro Rodríguez, A. (2003). Bosques y masas arboladas. En: Casares Long, J.J. Reflexiones sobre el medio ambiente en Galicia. Xunta de Galicia, Santiago de Compostela. 325-359

Ruiz de la Torre, J. (1979). Árboles y arbustos de la España peninsular. Fundación Conde del Valle de Salazar. Escuela Técnica Superior de Ingenieros de Montes, Madrid.

Verhulst, P.F. (1838). Notice sur la loi que la population pursuit dans son accroissement. Corresp. Math. Phys. 10.

Vignote Peña, S. \& Martínez Rojas, I. (2006). Tecnología de la madera. Mundi-Prensa, Madrid.

Vignote Peña, S., Picos Martín, J. \& Zamora Paniagua, R. (2000). Características de las principales maderas utilizadas en Bizkaia: tecnología y aplicaciones. Departamento de Agricultura de la Diputación Foral de Bizkaia, Bilbao.

Wagenführ, R. \& Scheiber, C. (1974). Holzatlas. Fachbuchverlag, Leipzig. 